Pacific Journal of Mathematic 


\section{ON CERTAIN TOEPLITZ OPERATORS IN TWO VARIABLES}

\section{STANLEy J. OSher}

The problem of inverting and/or factoring Weiner-Hopf operators in two variables is one of the basic unsolved problems in classical analysis. In this paper we shall consider operators which are a perturbation of a product of operators in one variable, the perturbation differing from such simple operators by an operator in one variable. The principal tools used are the spectral mapping theorem combined with the known results on operators in one variable.

2. Preliminaries. Consider the space $l_{2}$ of sequences of complex numbers

$$
\xi=\left\{\xi_{i j}\right\}_{i, j}^{\infty} c_{i j}=0
$$

with

$$
\|\xi\|=\left(\sum_{j=0}^{\infty} \sum_{k=0}^{\infty}\left|\xi_{k j}\right|^{2}\right)^{1 / 2}<\infty .
$$

Let

$$
a=\left\{a_{j}\right\}_{j=-\infty}^{\infty} \quad b=\left\{b_{j}\right\}_{j=-\infty}^{\infty} \quad c=\left\{c_{j}\right\}_{j=-\infty}^{\infty}
$$

be absolutely convergent sequences of complex numbers. Define

$$
\begin{aligned}
g & =\left\{g_{i j}\right\}_{i, j=-\infty}^{\infty} \\
g_{i j} & =a_{i} b_{j}+c_{j} \delta(j)
\end{aligned}
$$

for

$$
\delta(j)=0 \text { if } j \neq 0, \delta(0)=1 .
$$

It is clear that

$$
\sum_{j=-\infty}^{\infty} \sum_{i=-\infty}^{\infty}\left|g_{i j}\right|<\infty
$$

We are concerned with the operation

$$
\begin{gathered}
T_{g}: l_{2} \rightarrow l_{2}, \\
\left(T_{g} \xi\right)_{i j}=\sum_{L=0}^{\infty} \sum_{k=0}^{\infty} g_{j-k, j-L} \xi_{k L} \\
{\left[g_{i j}=g_{i, j}\right] .}
\end{gathered}
$$


Our techniques and results are exactly the same in the two variable Weiner-Hopf integral analogue.

Define

$$
\begin{aligned}
G\left(e^{i \theta}, e^{i \varphi}\right)=\sum_{j=-\infty}^{\infty} \sum_{k=-\infty}^{\infty} g_{k j} e^{-i j \varphi-i k \theta} \\
A\left(e^{i \theta}\right)=\sum_{k=-\infty}^{\infty} a_{k} e^{-i k \theta} \\
B\left(e^{i \varphi}\right)=\sum_{k=-\infty}^{\infty} b_{k} e^{-i k \varphi} \\
C\left(e^{i \varphi}\right)=\sum_{j=-\infty}^{\infty} c_{j} e^{-i j \varphi} .
\end{aligned}
$$

So

$$
G\left(e^{i \theta}, e^{i \varphi}\right)=A\left(e^{i \theta}\right) B\left(e^{i \varphi}\right)+C\left(e^{i \varphi}\right) .
$$

Let $\hat{l}_{2}$ denote the space of doubly infinite sequences in the second subscript, singly infinite in the first.

$$
\begin{aligned}
& \xi \in \tilde{l}_{2}, \quad \xi=\left\{\xi_{i j}\right\}_{j=-\infty, i=0}^{\infty} \\
& \|\xi\|_{\sim}=\left(\sum_{j=-\infty}^{\infty} \sum_{i=0}^{\infty}\left|\xi_{i j}\right|^{2}\right)^{1 / 2} .
\end{aligned}
$$

Let us Fourier transform $l_{2}$ and $\widetilde{l}_{2}$ with respect to the second subscript, i.e.,

$$
\hat{\xi}=\left\{\xi_{k}\left(e^{i \varphi}\right)\right\}_{k=0}^{\infty} \in E_{2}
$$

where

$$
\xi_{k}\left(e^{i \varphi}\right)=\sum_{j=0}^{\infty} \xi_{k j} e^{i j \varphi} .
$$

We shall obtain on $E_{2}$ a transformed Toeplitz operator whose elements are themselves Toeplitz operators

Define

$$
P_{+}^{(\varphi)} \sum_{j=-\infty}^{\infty} \xi_{k j} e^{i j \varphi}=\sum_{j=0}^{\infty} \xi_{k j} e^{i j \varphi},
$$

and on the space of singly semi-infinite sequences, define the Toeplitz map

$$
(A \eta)_{i}=\sum_{j=0}^{\infty} a_{i-j} \eta_{j} \cdot
$$

Thus, the total operator transforms to the compound operator

$$
P_{+}^{(\varphi)} B\left(e^{i \varphi}\right) A \hat{\xi}+P_{+}^{(\varphi)} C\left(e^{i \varphi}\right) \hat{\xi}=P_{+}^{(\varphi)} L\left(e^{i \varphi}\right) \hat{\xi} .
$$




\section{Main results.}

MaIN Theorem. $(\mathrm{A}) \Leftrightarrow(\mathrm{B}) \Leftrightarrow(\mathrm{C})$

(A) (1) $G\left(e^{i \theta}, e^{i \varphi}\right) \neq 0$ real $\theta, \varphi$.

(2) The change in argument of $G\left(e^{i \theta}, e^{i \varphi}\right)$ as $\theta$ goes from 0 to $2 \pi$ is 0 for any real $\varphi$.

(3) The change in argument of $G\left(e^{i \theta}, e^{i \varphi}\right)$ as $\varphi$ goes from 0 to $2 \pi$ is 0 for any real $\theta$.

(B) $L\left(e^{i \varphi}\right)$ can be factored

$L\left(e^{i \varphi}\right)=L_{-}\left(e^{i \varphi}\right) L_{+}\left(e^{i \varphi}\right)$ for $0 \leqq \varphi \leqq 2 \pi$ where

$L_{-}\left(e^{i \varphi}\right), L_{+}\left(e^{i \varphi}\right)$ commute and are continuous in $\varphi$ and bounded for each $\varphi$. Moreover $L_{-}\left(e^{i \varphi}\right)$ has an analytic operator valued extension to $|z|>1$ which is invertible for these $z, L_{+}\left(e^{i \varphi}\right)$ has an analytic operator valued extension to $|z|<1$ invertible for these $z$. This factorization is unique if $L_{-}(\infty)=I$.

(C) $T_{g}$ is invertible.

Proof. Assume (A). Consider

$$
\mu B\left(e^{i \varphi}\right)+C\left(e^{i \varphi}\right)
$$

where first $\mu=A\left(e^{i \theta_{0}}\right)$ for some real $\theta_{0}$. Conditions (1) and (3) and the results of [1] guarantee that a factorization

$$
\mu B\left(e^{i \varphi}\right)+C\left(e^{i \varphi}\right)=D_{-}\left(\mu, e^{i \varphi}\right) D_{+}\left(\mu, e^{i \varphi}\right)
$$

exists for each such $\mu$ where the factors $D_{-}$and $D_{+}$have the same properties as functions as $L_{-}\left(e^{i \varphi}\right)$ and $L_{+}\left(e^{i \varphi}\right)$ have as operators. Since property 3 is a homotopic invariant, such a factorization fails to exist for some $\mu$ in the spectrum of $A$ if and only if $\exists \mu_{0}$ in the spectrum of $A$ and some real $\varphi_{0}$ with

$$
\mu_{0} B\left(e^{i \varphi_{0}}\right)+C\left(e^{i \varphi_{0}}\right)=0 .
$$

If $B\left(e^{i \varphi_{0}}\right) \neq 0$, then $C\left(e^{i \varphi_{0}}\right) \neq 0$ by condition (1). Thus,

$$
\mu_{0}=-\frac{C\left(e^{i \varphi_{0}}\right)}{B\left(e^{i \varphi_{0}}\right)}
$$

But by condition (2) the change in argument as $\theta$ goes from 0 to $2 \pi$ of $\left[a\left(e^{i \theta}\right)-\mu_{0}\right]=0$, thus $\mu_{0}$ does not belong to the spectrum of $A$.

Thus the factorization (3.4) exists for all $\mu$ in the spectrum of $A$. It is clear from the construction involved in [1] that the factors are locally analytic in $\mu$ for $\mu$ in the spectrum of $A$. 
We normalize so that $D_{-}(\mu, \infty)=1$. Then the equation (for any $\mu$ in the spectrum of $A$ )

$$
P_{+}^{(\varphi)}\left[\mu B\left(e^{i \varphi}\right)+C\left(e^{i \varphi}\right)\right] h_{\mu}\left(e^{i \varphi}\right)=1,
$$

on the space of Fourier transforms of semi-infinite sequences with one subscript, has the unique solution

$$
h_{\mu}\left(e^{i \varphi}\right)=D_{+}^{-1}\left(\mu, e^{i \varphi}\right) .
$$

Single-valuedness of the factors is now immediate. Thus each factor is analytic separately in $\mu$ on the spectrum of $A$. Moreover, for such $\mu, D_{-}\left(\mu, e^{i \varphi}\right)$ has an analytic extension to $|z|>1$, invertible for $|z| \geqq 1$. Thus, the operator $D_{-}\left(A, e^{i \varphi}\right)$ has the same properties, by the spectral mapping theorem. We may make the analogous statements about $D_{+}\left(\mu, e^{i \varphi}\right)$.

Thus, by the spectral mapping theorem we may replace $\mu$ by $A$ in $D_{-}\left(\mu, e^{i \varphi}\right), D_{+}\left(\mu, e^{i \varphi}\right)$ and obtain $L_{-}\left(e^{i \varphi}\right), L_{+}\left(e^{i \varphi}\right)$ with all the appropriate properties of analyticity in $z$ and invertibility.

Next, suppose

$$
M_{-}\left(e^{i \varphi}\right) M_{+}\left(e^{i \varphi}\right)=L_{-}\left(e^{i \varphi}\right) L_{+}\left(e^{i \varphi}\right),
$$

$M_{-}, M_{+}$having the same properties, then

$$
L_{-}^{-1}\left(e^{i \varphi}\right) M_{-}\left(e^{i \varphi}\right)=L_{+}\left(e^{i \varphi}\right) M_{+}^{-1}\left(e^{i \varphi}\right)
$$

and hence they are both analytic in the whole plane and equal to the identity at $\infty$, or

$$
L_{-}\left(e^{i \varphi}\right)=M_{-}\left(e^{i \varphi}\right), \quad L_{+}\left(e^{i \varphi}\right)=M_{+}\left(e^{i \varphi}\right)
$$

or the factorization is unique.

Thus $A \Rightarrow B$.

Now, let us assume $B$. We wish to solve:

$$
P_{+}^{(\varphi)} L\left(e^{i \varphi}\right) \hat{\xi}=\hat{\eta} .
$$

Consider the operator $P_{+}^{(\varphi)} L_{-}^{-1}\left(e^{i \varphi}\right)$.

By the isometry of the Fourier transform

$$
\left\|P_{+}^{(\varphi)} L_{-}^{-1}\left(e^{i \varphi}\right)\right\| \leqq \sup _{0 \leqq \varphi \leqq 2 \pi}\left\|\left|L_{-}^{-1}\left(e^{i \varphi}\right)\right|\right\|
$$

where || $\mid$ || denotes operator norm on the space of semi infinite sequences with one subscript. Similarly

$$
\left\|L_{+}^{-1}\left(e^{i \varphi}\right)\right\| \leqq \sup _{0 \leqq \varphi \leqq 2 \pi}\left\|\mid L_{+}^{-1}\left(e^{i \varphi}\right)\right\| \| .
$$


Let

$$
\hat{\xi}=L_{+}^{-1}\left(e^{i \varphi}\right) P_{+}^{(\varphi)} L_{-}^{-1}\left(e^{i \varphi}\right) \hat{\eta}
$$

Then

$$
P_{+} L\left(e^{i \varphi}\right) \hat{\xi}=\hat{\eta}+P_{+}^{(\varphi)} L_{-}\left(e^{i \varphi}\right)\left(P_{+}^{(\varphi)}-I\right) L^{-1}\left(e^{i \varphi}\right) \hat{\eta},
$$

but by the anti-analyticity of $L_{-}\left(e^{i \varphi}\right)$ and the definition of $P+^{(\varphi)}$ we have

$$
P_{+}^{(\varphi)} L\left(e^{i \varphi}\right) \hat{\xi}=\hat{\eta}
$$

Thus $T_{g}$ has a right inverse. This right inverse is easily shown to be a left inverse using the anti-analyticity of $L_{-}^{-1}\left(e^{i \varphi}\right)$.

Next we assume $C$. Suppose $G\left(e^{i \theta_{0}}, e^{i \varphi_{0}}\right)=0$.

Then, if $T_{g}$ is invertible, so is $T_{g_{M}}+\delta I$, for all $M$ large enough and all $|\delta|$ small enough and:

$$
G_{M}\left(e^{i \theta}, e^{i \varphi}\right)=\sum_{j=-M}^{M} a_{j} e^{-i j \theta} \sum_{k=-M}^{M} b_{k} e^{-i k \varphi}+\sum_{k=-M}^{M} b_{k} e^{-i k \varphi}
$$

Moreover, we may choose $M_{0}, \varphi_{1}, \theta_{1}, \delta_{0}$ such that $\left|\delta_{0}\right|<\delta$ and $M_{0} \geqq M$ and

$$
G_{M_{0}}\left(e^{i \theta_{1}}, e^{i \varphi_{1}}\right)+\delta_{0}=0 .
$$

Next consider the sequence of vectors $\xi^{N}$, where

$$
\begin{aligned}
& \xi_{j k}^{N}=\frac{1}{\sqrt{N+1}} e^{i\left(j \theta_{1}+k \varphi_{1}\right)} \text { if } 0 \leqq j, k \leqq N \\
& \xi_{j k}^{N}=0 \text { otherwise } .
\end{aligned}
$$

Clearly

$$
\left\|\xi^{N}\right\|=1 \text { while } \lim _{N \rightarrow \infty}\left(T_{g_{M_{0}}}+\delta_{0} I\right) \xi^{N}=0 .
$$

Contradiction.

Now suppose the change in argument in condition 2 is $2 \pi \eta_{\theta} \neq 0$. (This number is obviously independent of $\varphi$ ). Thus, for $M$ large, $G_{M}\left(e^{i \theta}, e^{i \varphi}\right)$ has the same $\eta_{\theta}$ for each $\varphi_{0}$. If $\eta_{\theta}<0$, then $L_{M}\left(e^{i \varphi_{0}}\right)$ annihilates some vector

$$
K=\left\{k_{0}, k_{1}, \cdots\right\}, \quad\|K\|=1 .
$$

But then the sequence

$$
\begin{aligned}
& \xi_{k j}^{N}=\frac{1}{\sqrt{N+1}} k_{i} e^{i j \varphi_{0}} \text { if } 0 \leqq j \leqq N \\
& \xi_{i j}^{N}=0 \text { if } N<j, \text { has the property }
\end{aligned}
$$




$$
\left\|\xi^{N}\right\|=1 \text {, but } \lim _{N \rightarrow \infty} T_{g_{M}} \xi^{N}=0 \text {. }
$$

Thus $T_{g_{M}}$ is not invertible, hence neither is $T_{g}$. If $\eta_{\theta}>0$, we merely consider $T_{g}^{*}$. Finally, we assume that the change in argument is $2 \pi \eta_{\varphi} \neq 0$ in condition (3). Then consider

$$
e^{-i \eta \varphi \varphi} G\left(e^{i \theta}, e^{i \varphi}\right)=H\left(e^{i \theta}, e^{i \varphi}\right) \text {. }
$$

This function obeys the conditions of (A), hence it is factorable and $\mathrm{T}_{h}$ is invertible. However, if $\eta_{\varphi}>0$, then

$$
S^{* \eta_{\varphi}}=S_{\varphi}^{* \eta_{\varphi}} T_{g}\left(T_{g}\right)^{-1}=T_{h}\left(T_{g}\right)^{-1}
$$

where $S_{\varphi}$ is the right shift operator on the $j$ subscript. This is impossible since the two operators on the right are invertible. If $\eta_{\varphi}<0$, we merely consider the adjoint.

Thus $(C) \Rightarrow(A)$, and we are finished.

4. Example. Let $B\left(e^{i \varphi}\right)$ and $C\left(e^{i \varphi}\right)$ have finite expansions

$$
\begin{aligned}
& B=\sum_{j=-N}^{M} b_{j} e^{-i j \varphi} \\
& C=\sum_{j=-N}^{M} c_{j} e^{-i j \varphi}
\end{aligned}
$$

and suppose

$$
\mu b_{-N}+c_{-N} \neq 0 \text { for } \mu \text { in the spectrum of } A .
$$

Assume conditions 1, 2, 3. Then we may factor

$$
\mu B(z)+C(z)=\left(\mu b_{-N}+c_{-N}\right) \prod_{i=1}^{N}\left(z-x_{i}(\mu)\right) \prod_{j=1}^{M}\left(1-\frac{y_{j}^{(\mu)}}{z}\right)
$$

for all $\mu$ in the spectrum of $A$ and each $\left|x_{i}(\mu)\right|>1,\left|y_{j}(\mu)\right|<1$. See [3]. Then it follows that

$$
L(z)=L_{-}(z) L_{+}(z)
$$

with

$$
\begin{aligned}
& L_{-}(z)=\prod_{j=1}^{M}\left(1-\frac{y_{j}^{M}(A)}{z}\right) \\
& L_{+}(z)=\left(A b_{-N}+c_{-N}\right) \prod_{i=1}^{N}\left(z-x_{i}(A)\right) .
\end{aligned}
$$

We expect this factorization to play an important role in the study of difference equations arising from hyperbolic systems in regions in space having corners. 


\section{BIBLIOGRAPHY}

1. A. Calderon, F. Spitzer, and H. Widom, Inversion of Toeplitz matrices, Illinois J. Math. 3 (1959), 490-498.

2. M. G. Krein, Integral equations on the half-line with a difference kernel, Uspehi Mat. Nauk 13 (1958), 3-120.

3. S. J. Osher, Systems of difference equations with general homogeneous boundary conditions, Amer. Math. Soc. Trans. 137 (1969), 177-201.

Received June 9, 1969.

UNIVERSITY OF CALIFORNIA, BERKELEY 



\section{PACIFIC JOURNAL OF MATHEMATICS}

EDITORS

H. SAMELSON

Stanford University

Stanford, California 94305

RichaRd PIERCE

University of Washington

Seattle, Washington 98105
J. DUGUNDJI

Department of Mathematics

University of Southern California

Los Angeles, California 90007

RICHARD ARENS

University of California

Los Angeles, California 90024

\section{ASSOCIATE EDITORS}

E. F. BECKENBACH

B. H. NeumanN

F. WOLF

K. YOSHIDA

\section{SUPPORTING INSTITUTIONS}

UNIVERSITY OF BRITISH COLUMBIA

CALIFORNIA INSTITUTE OF TECHNOLOGY

UNIVERSITY OF CALIFORNIA

MONTANA STATE UNIVERSITY

UNIVERSITY OF NEVADA

NEW MEXICO STATE UNIVERSITY

OREGON STATE UNIVERSITY

UNIVERSITY OF OREGON

OSAKA UNIVERSITY

UNIVERSITY OF SOUTHERN CALIFORNIA
STANFORD UNIVERSITY

UNIVERSITY OF TOKYO

UNIVERSITY OF UTAH

WASHINGTON STATE UNIVERSITY

UNIVERSITY OF WASHINGTON

AMERICAN MATHEMATICAL SOCIETY CHEVRON RESEARCH CORPORATION TRW SYSTEMS

NAVAL WEAPONS CENTER 


\section{Pacific Journal of Mathematics}

May, 1970

Johan Aarnes, Edward George Effros and Ole A. Nielsen, Locally compact

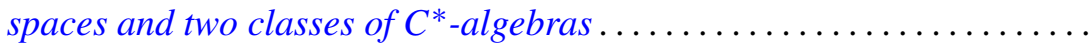

Allan C. Cochran, R. Keown and C. R. Williams, On a class of topological

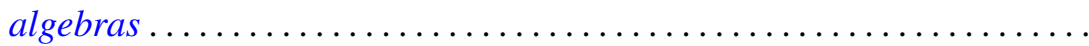

John Dauns, Integral domains that are not embeddable in division rings ....

Robert Jay Daverman, On the number of nonpiercing points in certain

crumpled cubes.....................................

Bryce L. Elkins, Characterization of separable ideals ................

Zbigniew Fiedorowicz, A comparison of two naturally arising uniformities

on a class of pseudo-PM spaces ...........................

Henry Charles Finlayson, Approximation of Wiener integrals of functionals

continuous in the uniform topology ........................

Theodore William Gamelin, Localization of the corona problem ...........

Alfred Gray and Paul Stephen Green, Sphere transitive structures and the

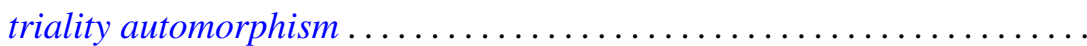

Charles Lemuel Hagopian, On generalized forms of aposyndesis ..........

J. Jakubík, On subgroups of a pseudo lattice ordered group ...............

Cornelius W. Onneweer, On uniform convergence for Walsh-Fourier

series..................................

Stanley Joel Osher, On certain Toeplitz operators in two variables ...

Washek (Vaclav) Frantisek Pfeffer and John Benson Wilbur, On the

measurability of Perron integrable functions............

Frank J. Polansky, On the conformal mapping of variable regions...

Kouei Sekigawa and Shûkichi Tanno, Sufficient conditions for a Riemannian manifold to be locally symmetric ...................

James Wilson Stepp, Locally compact Clifford semigroups ....

Ernest Lester Stitzinger, Frattini subalgebras of a class of solvable Lie

algebras ................................

George Szeto, The group character and split group algebras...

Mark Lawrence Teply, Homological dimension and splitting torsion

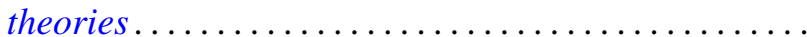

David Bertram Wales, Finite linear groups of degree seven. II ...

Robert Breckenridge Warfield, Jr., An isomorphic refinement theorem for

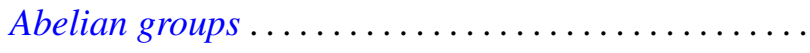

James Edward West, The ambient homeomorphy of an incomplete subspace

of infinite-dimensional Hilbert spaces................

Peter Wilker, Adjoint product and hom functors in general topology ...

Daniel Eliot Wulbert, A note on the characterization of conditional 